\title{
Pseudoactinomycotic radiate granules in the maxillary sinus. A case report
}

\author{
Ivo Starek ${ }^{\mathrm{a}}$, Jaroslav Horacek ${ }^{\mathrm{b}}$, Richard Salzman ${ }^{\mathrm{a}}$
}

\begin{abstract}
Background. Pseudoactinomycotic radiate granules are biologically inactive formations simulating true actinomycotic granules. They occur mainly in the female genital tract. Here we describe a previously unreported case of these granules in a maxillary sinus.

Methods and Results. A 74-year old man underwent maxillectomy for a carcinoma of the right alveolar ridge. In the tumor-free sinus mucosa, a deposit of condensed mucus, cell debris and eosinophilic granules, surrounded by leukocytes, were seen. These Gram-positive granules were refractile, not bi-refringent and revealed radiate pattern with peripheral club-like thickening. Alcian blue staining was negative, PAS reaction was present at the periphery. ZiehlNeelsen, Grocott and Kongo red reactions tested negatively. In the mucoid surroundings, strong IgM, moderate lgG, weak $\lg A$ and $\lg G 4$, but no $\lg D$ immunoreactivity was present.
\end{abstract}

Conclusions. PAMRAGs in the maxillary sinus have not been described so far. Despite their extremely rare occurence, they must be taken into consideration in histopathological differential diagnosis of actinomycosis of that anatomic location.

Key words: maxillary sinus, pseudoactinomycotic granules, Splendor-Hoeppli phenomenon

Received: February 28, 2019; Accepted: March 28, 2019; Available online: April 17, 2019

https://doi.org/10.5507/bp.2019.012

(c) 2020 The Authors; https://creativecommons.org/licenses/by/4.0/

${ }^{a}$ Department of Otorhinolaryngology and Head and Neck Surgery, Faculty of Medicine and Dentristry, Palacky University Olomouc and University Hospital Olomouc, Czech Republic

${ }^{b}$ Department of Clinical and Molecular Pathology, Faculty of Medicine and Dentristry, Palacky University Olomouc and University Hospital Olomouc, Czech Republic

Corresponding author: Richard Salzman, e-mail: richard.salzman@fnol.cz

\section{INTRODUCTION}

Pseudoactinomycotic radiate granules (PAMRAGs) are eosinophilic formations composed of club-like rods simulating actinomycotic granules. In human beings, they occur mostly in the female genital tract where they are typically, but not exclusively, associated with the use of intrauterine contraceptive device (IUCD) $\left(\right.$ ref. $\left.^{1-3}\right)$. In extragenital locations, they were described in colloid cysts of the third ventricle of the CNS (ref. ${ }^{4}$ ) and inspissated bronchial mucus 5 . Genesis of the PAMRAGs remains obscure. In IUCD users, they might represent particles of that device or organic deposits precipitated on its fragments. In extragenital locations, they could be an antigen-antibody complex (also called as Splendor-Hoeppli phenomenon). However, non-immune mechanisms are also supposed to be responsible for the formation of these granules. Here we report a case of PAMRAGs in the maxillary sinus and discuss the nature and comment on their clinical relevance. To the best of our knowledge, this the first description of these granules at that site.

\section{CASE REPORT}

A 74-year old man was diagnosed with a T2N3MX squamous cell carcinoma of the alveolar ridge of his right upper jaw. The treatment consisted of a partial maxillec- tomy, comprehensive I-V neck dissection and subsequent radiotherapy. Histopathologic analysis of the surgical specimen revealed no presence of a carcinoma in the maxillary sinus, the mucosa of which showed focally regressive inflammatory changes with sparsely distributed polymorphonuclear leukocytes. Condensed mucus contained cell debris and widely spreading, usually spheric eosinophilic radiate granules surrounded by leukocytes (Fig. 1). Less often, some hyphal-like granules were present (Fig. 2). The PAMRAGs were refractile but not birefringent. In Gram staining, they revealed peripheral club-like thickening (Fig. 3). Alcian blue staining was negative, weak PAS reaction was stronger at the periphery. Ziehl-Neelsen, Grocott and Kongo red reactions tested negatively. In the mucoid substance, precipitates revealing strong IgM, moderate IgG, weak IgA and IgG4, but no IgD immunoreactivity, were present. The granules showed conspicuous CD68 positivity, which was faint in their surroundings.

\section{DISCUSSION}

Pathomorphology of PAMRAGs closely resembles that of true actinomycotic granules. However, there are some distinct features enabling their differentiation. The latter are non-refractile formations with an eosinophilic granular dense centre and peripheral Gram, ZiehlNeelsen and PAS-positive, thin (circa $1 \mu \mathrm{m}$ ) branching 


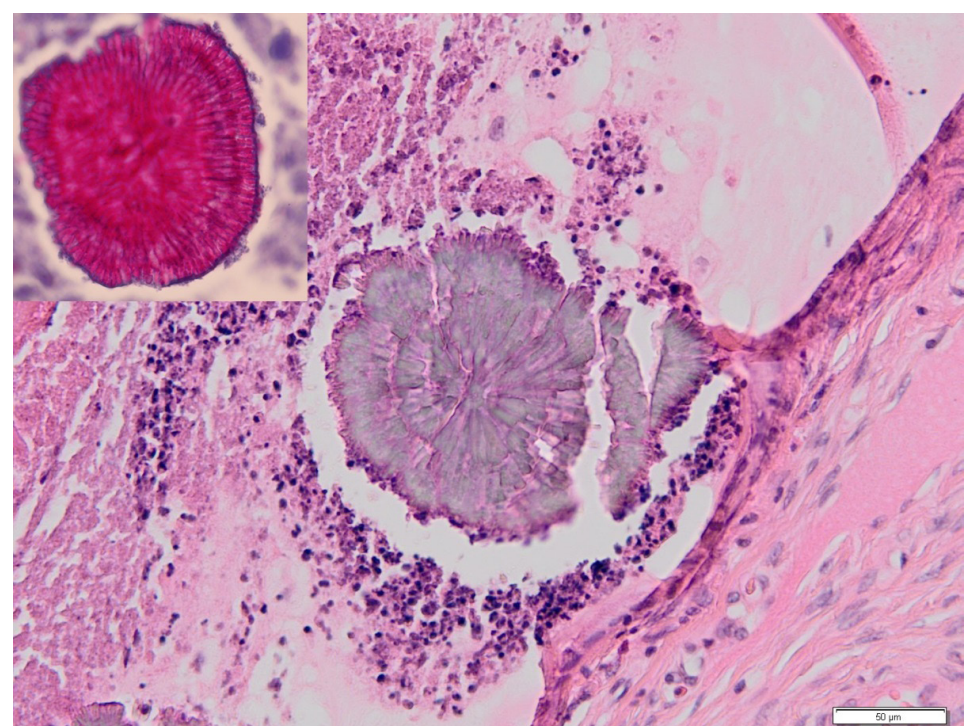

Fig. 1. A pseudoactinomytic granule in a pool of mucus is surrounded by leukocytes and cellular debris $(\mathrm{HE}, \times 400)$.

Inset: At high magnification (x1000), distinct radiate pattern of a PAMRAG is evident.

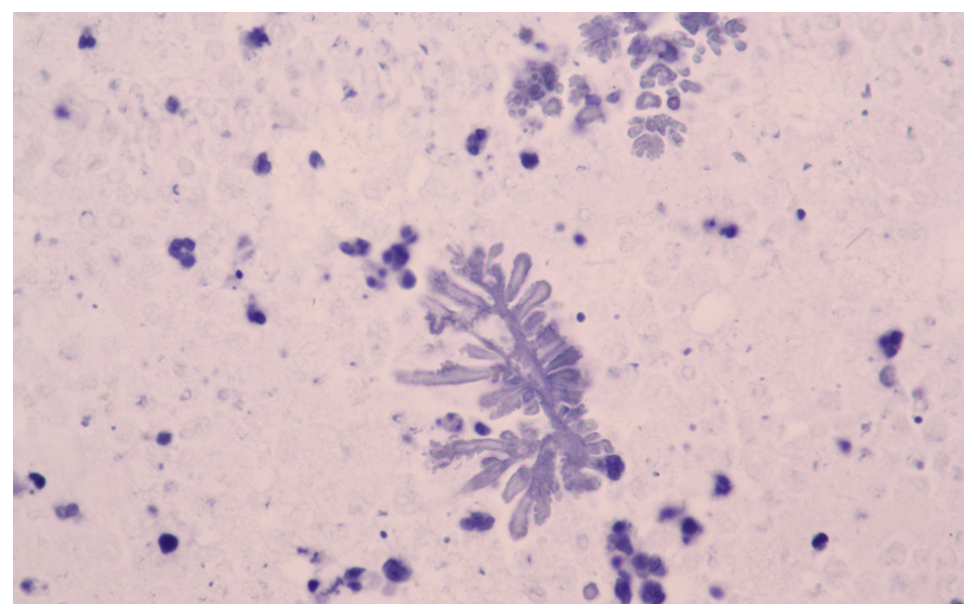

Fig. 2. A hyphal-like PAMRAG in mucoid substance $(\mathrm{HE}, \times 400)$.

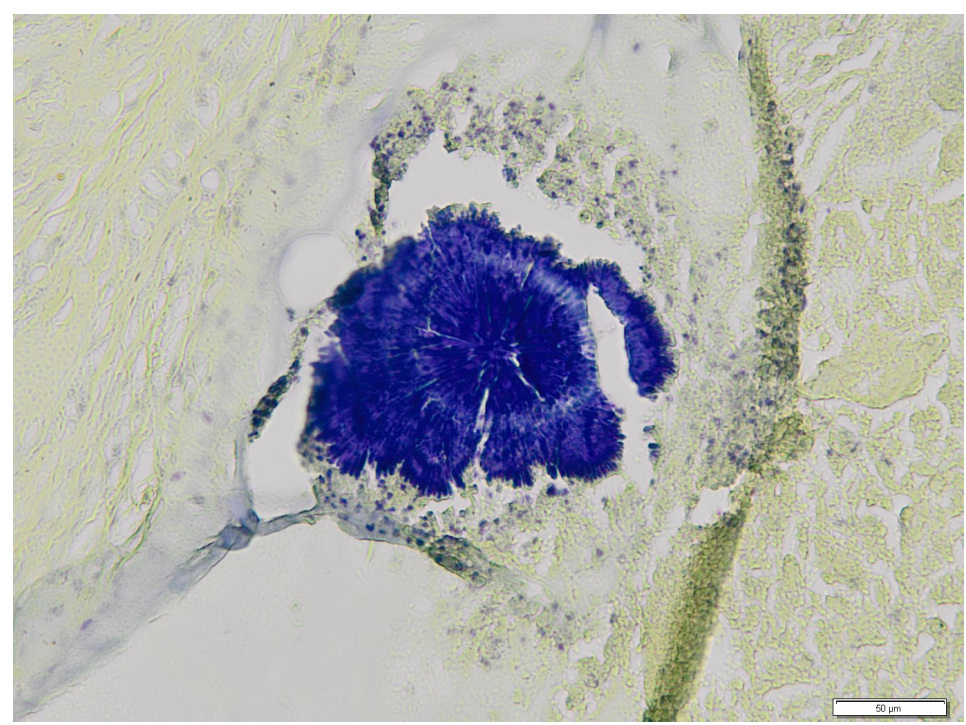

Fig. 3. Strongly Gram-positive radiating granules reveal peripheral clubbing (arrows) $(\times 400)$. 
filaments. On the contrary, central core lacking refractile PAMRAGs show thick (> $10 \mu \mathrm{m})$ irregular non-branching club-like peripheral projections. They occasionally reveal histochemical profile similar to that in true actinomycotic granules ${ }^{3,6,7}$. In these cases the differential diagnosis is based on the presence of actinomycotic filaments, highlighted by Gomori reaction.

The nature and morphogenesis of PAMRAGs has not been satisfactorily elucidated yet. In many extragenital locations, primarily in veterinary fields, radiating eosinophilic deposits (occasionally referred to as Splendor-Hoeppli phenomenon) were found to surround certain bacteria, fungi and parasites or inorganic materials. By some authors these granules were considered to be a non-specific reaction to these agents and disintegrated surrounding reactive cells ${ }^{8,9}$. Smith et al. ${ }^{10}$ and von Lichtenberg et al. ${ }^{11}$ noticed similarities in the histochemistry of schistoma eggs and associated Splendor-Hoeppli phenomenon. They concluded that the phenomenon represented an immune-host reaction to antigenic products of cephalic glands of that parasite, resulting in the formation of an antigen-antibody complex precipitating around the eggshells. That opinion was in concert with an ultrastructural study demonstrating that the substance of a fungiassociated Splendor-Hoeppli phenomenon was composed of cytoplasmic and nuclear debris derived from reactive cells and amorphous granular material considered to be antigen-antibody complex ${ }^{12}$.

In the female genital tract, neither specific stainings nor ultrastructural analysis could demonstrate presence of any infection agents in the PAMRAGs (ref., ${ }^{2,3,7}$ ). O 'Brien et al. initially believed that the PAMRAGs represented particles of plastic IUCD dissociated in the endometrium owing to cyclically changing environment of the endometrial cavity ${ }^{13}$. In their later study the authors analyzed ultrastructure of copper IUCD-associated pseudoactinomycotic granules identified in histologic sections of an uterine curetting taken from a female patient ${ }^{2}$. Neither biological nor artificial materials could be found in the granules, which - as well as their immediate vicinity contained various chemical elements, primarily copper. Therefore it was concluded that the PAMRAGS were precipitates of the latter element, which could have been a derivative of metallic component of the IUCD or endogenous copper, which is abundant in lipofuscin. The authors also questioned their previous view on the plastic character of the PAMRAGs. Striepecke et al. ${ }^{7}$ studied pseudoactinomycotic granules found in 14 of 123 uterine curettage specimens in women using copper compounded IUCD. In the granules, empty areas were seen. The authors considered them to be negative imprints of IUCDassociated metallic particles. In one case, the surface of the removed IUCD was covered with an amorphous hyaline mass, revealing radial pattern. This finding suggested that the pseudogranules were organic deposits on copper fragments detached from the IUCD.

The fact, that pseudomycotic granules can occur in tissues free of any artificial materials, raises the question of their endogenous, presumably cellular origin.

Paedberg et al. ${ }^{3}$ found marginal IgG (but not IgD, IgA and IgM) positivity in pseudoactinomycotic granules present in a bacteria-free uterine curettage specimen taken from an IUCP user. At the border of the pseudogranules, granulocytes and signs of intensified phagocytosis were present. The authors concluded that the PAMRAGs consisted chiefly of products of disintegration of macrophages and immunoglobulin with added debris and fibrin. In our case, in which neither infectious agents nor any non-biological particles could be demonstrated, the PAMRAGs showed positive CD68 reaction and were surrounded by leukocytes and immunoglobulin precipitates. These findings suggest that immunological mechanisms, similar to those hypothesized in the Splendor-Hoepli phenomenon, were involved in the pathogenesis of the granules composed of PAS positive mucus and leukocytic debris.

On the other side, Bhagavan et al. ${ }^{1}$ studied 6 cases of genital tract PAMRAGs, three of which were not associated with the IUCD. In all samples, histochemical analysis identified lipids, calcium, neutral glycoproteins, but no fibrin, immunoglobulins or complement. The authors concluded that the PAMRAGs were not formed by an immune process, but derived from host leukocytes aggregated in a non-specific response to IUCD or bacteria present in that location.

Powers et al. ${ }^{4}$ analyzed 3 cases of colloid cysts originating in the third ventricle. They contained eosinophilic, hyphal-like structures. The light microscopy, histochemistry and ultrastructure corresponded with the PAMRAGs and failed to demonstrate presence of any bacterial, actinomycotic or fungal agents. Special stains suggested that these hyphal-like structures represented admixture of the material of degenerating nuclei and cytoplasmatic membranes of the lining epithelial cells.

The PAMRAGs are biologically non-active formations. Their only clinical relevance thus resides in that they might be misdiagnosed as a true actinomycosis. That infection, showing usually low pathogenicity is seldom in the cervicofacial region and exceptionally rare in the paranasal sinuses ${ }^{14,15}$. However, in the latter locations it may take a very invasive course, causing even intracranial complications. Therefore, especially immunodeficient patients diagnosed with paranasal sinus actinomycosis should be started on a long-term antibiotic therapy postoperatively, which would be unnecessary in cases of PAMRAGs.

\section{CONCLUSIONS}

PAMRAGs are harmless pathomorphological formations, the nature of which remains unclear. They are encountered mostly in the female genital tract. In the paranasal sinuses, their occurence is extremely rare. Nonetheless, also in this location they must be taken into consideration in the histopathological differential diagnosis of actinomycosis to avoid unnecessary antibiotic therapy.

Acknowledgement: This work was supported by Ministry of Health of the Czech Republic research grants [16- 
31881A, DRO FNO1, 00098892], and Internal grant of Palacky University [IGA LF 2018-006]. We are grateful to Ing. Mgr. Ivo Úberall, PhD. (Department of Clinical and Molecular Pathology, Faculty of Medicine and Dentristry, Palacký University Olomouc, Czech Republic) for his technical assistance for preparing the manuscript.

Author contributions: IS: literature search, manuscript writing; JH: review of the manuscript, histological assessment; RS: review of the manuscript, final approval.

Conflict of interest statement: The authors state that there are no conflicts of interest regarding the publication of this article.

\section{REFERENCES}

1. Bhagavan BS, J. Ruffier J, Shinn B. Pseudomycotic radiate granules in the lower female genital tract: relationship to Splendor-Hoeppl phenomenon. Hum Pathol 1982;13:898-904.

2. O'Brien PK, Lea PJ, Roth-Moyo LA. Structure of a radiate pseudocolony associated with an intrauterine contraceptive device. Hum Pathol 1985;16:1153-6.

3. Paedberg BB, Kolb SA, August C. S. Schroder. IUD-associated pseudoactinomycotic radiate granules (Spendore-Hoeppli phenomenon) in uterine curettage. Pathology 2002;23:135-9.
4. Powers JM, Dodds HM. Primary actinomycosis of the third ventricle - the colloid cyst. A histochemical and ultrastructural study. Acta Neuropathol 1977;37:21-6.

5. O'Brien PK, Roth-Moyo LA. Letter to the editor. Hum Pathol $1983 ; 14: 829$.

6. Pritt B, Mount SL, Cooper K, H. Blaszyk. Pseudoactinomycotic radiate granules of the gynaecological tract: review of a diagnostic pitfall. J Clin Pathol 2009;62:1123-6.

7. Striepecke E, Bollmann R. Pseudo-sulphur granules (pseudo-actinomycotic granules) in intrauterine device patients. Geburtshilfe Frauenheilkd 1994;51:171-3.

8. Moore M. Radiate formation on pathogenic fungi in human tissue. Arch Pathol 1946;42:113-53.

9. Liber AF, Choi HS. Splendor-Hoeppli phenomenon about silk sutures in tissue. Arch Pathol 1973;95:217-20.

10. Smith JH, Lichtenberg F. The Hoeppli phenomenon in schistosomiasis; histochemistry. Am J Pathol 1967;50:993-1001.

11. Lichtenberg F, Smith JH, Cheever AW. The Hoeppli phenomenon in schistosomiasis; comparative pathology and immunopathology. Am J Trop Med 1966;15:886-95.

12. Williams $A O$, Lichtenberg $F$, Smith $J H$, Martinson FD. Ultrastructure of phycomycosis due to Entomophthora, basidiobolus, and associated "Splendore-Hoeppli" phenomenon. Arch Pathol 1969;87:459-68.

13. O’Brien P, Roth-Moyo LA, Davis BA. Pseudosulphur granules associated with intrauterine contraceptive devices. Am J Clin Pathol 1981;75:822-5.

14. Roth M, Montone LT. Actinomycosis of the paranasal sinuses: A case report and review. Otolaryngol Head Neck Surg 1996;114:818-21.

15. Sánchez Legaza E, Cercera OC, Caravillo JIM. Actinomycosis of the paranasal sinuses. Acta Otorrinolaryngol Esp 2013;64:310-11. 